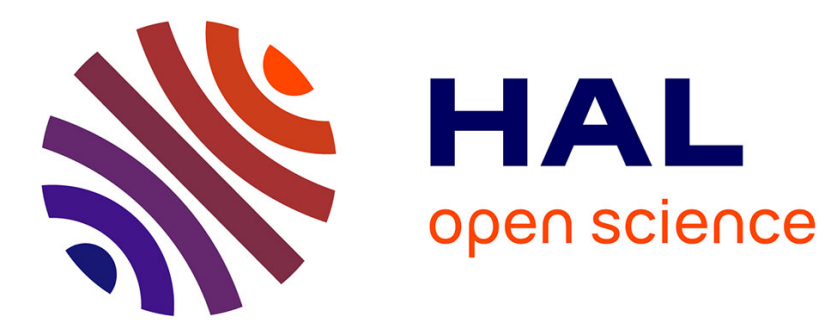

\title{
Saliency based visualization of hyper-spectral images
}

Haris Ahmad Khan, Muhammad Murtaza Khan, Khurram Khurshid, Jocelyn

Chanussot

\section{To cite this version:}

Haris Ahmad Khan, Muhammad Murtaza Khan, Khurram Khurshid, Jocelyn Chanussot. Saliency based visualization of hyper-spectral images. IGARSS 2015 - IEEE International Geoscience and Remote Sensing Symposium, Jul 2015, Milan, Italy. pp.1096-1099, 10.1109/IGARSS.2015.7325961 . hal-01672533

\section{HAL Id: hal-01672533 https://hal.science/hal-01672533}

Submitted on 5 Jan 2018

HAL is a multi-disciplinary open access archive for the deposit and dissemination of scientific research documents, whether they are published or not. The documents may come from teaching and research institutions in France or abroad, or from public or private research centers.
L'archive ouverte pluridisciplinaire HAL, est destinée au dépôt et à la diffusion de documents scientifiques de niveau recherche, publiés ou non, émanant des établissements d'enseignement et de recherche français ou étrangers, des laboratoires publics ou privés. 


\title{
SALIENCY BASED VISUALIZATION OF HYPER-SPECTRAL IMAGES
}

\author{
Haris Ahmad Khan ${ }^{1}$, Muhammad Murtaza Khan ${ }^{2,3}$, Khurram Khurshid ${ }^{1}$, Jocelyn Chanussot ${ }^{4}$ \\ ${ }^{1}$ EE Department, Institute of Space Technology, 1, Islamabad highway, Islamabad, 44000, Pakistan \\ ${ }^{2}$ SEECS, National University of Sciences and Technology, Islamabad, 44000, Pakistan \\ ${ }^{3}$ Faculty of Computing and Information Technology, University of Jeddah, Jeddah, Saudi Arabia \\ ${ }^{4}$ GIPSA-Lab, Grenoble University, Grenoble, France \\ ${ }^{1}\left\{\right.$ haris.ahmad02, khurram.khurshid\} @ist.edu.pk, ${ }^{2,3}$ muhammad.murtaza@ seecs.edu.pk, \\ jjocelyn.chanussot@gipsa-lab.grenoble-inp.fr
}

\begin{abstract}
The problem with visualization of hyper-spectral images on tri-stimulus displays arises from the fact that they contain hundreds of spectral bands while generally used display devices support only three bands/channels namely blue, green and red. Therefore, for visualization a hyper-spectral (HS) image has to be reduced to three bands. The main challenge while performing this band reduction is to retain and display the maximum information available in a hyper-spectral image. Human visual system focuses attention on certain regions in images called "salient regions". Therefore to provide a comprehensive representation of hyper-spectral data on tri-stimulus displays we propose to use a weighted fusion method of saliency maps and hyper-spectral bands. The efficacy of the proposed algorithm has been demonstrated by tests on both urban and countryside images of AVIRIS and ROSIS sensors.
\end{abstract}

Index Terms - Hyperspectral Images, Saliency, Human Visual System, Visualization

\section{INTRODUCTION}

Hyper-spectral images may comprise of tens to hundreds of channels. Visualization of such images is a challenge because each channel may contain information that is necessary for completely displaying the constituents of the image. Existing visualization schemes are limited in their capability to visualize hyper-spectral images because they either select three channels out of the available large number of spectral channels (e.g. Principal Component Analysis (PCA) based visualization) or they do not give different weightages to different prominent objects based upon their spatial characteristics (e.g. Color Matching Function (CMF) stretching).
The shortcoming of PCA based dimensionality reduction and visualization schemes is loss of spectral information because selection of principal components is based on variance and their spectral position is not taken into account. Also, these schemes do not incorporate any information related to Human Visual System (HVS) while performing dimensionality reduction. Human visual attention assists our capability to rapidly locate the most significant information in an image or a scene [1]. It is generally presumed that "salient" regions in images draw more attention than other parts of the image. Therefore, it is desirable to guide the dimensionality reduction process by taking into consideration the human visual system when displaying a hyper-dimensional data on a tri-dimensional (tri-stimulus) rendering system.

Color Matching Function (CMF) performs dimensionality reduction based upon a linear combination of the $r(\lambda), g(\lambda)$ and $b(\lambda)$ wavelengths. The shortcoming of CMF based channel reduction scheme is that it does not take into account any information about spatial features in an image and hence objects or regions with approximately similar spectral pattern may not be distinguishable in the final visualization.

A solution to the above-defined issues is to use saliency information of images during visualization as proposed in [2]. However, the authors propose to generate a single channel i.e. monochromatic image from hyper-spectral channels and then determine the salient regions in the monochromatic image. The issue with this approach is that objects or regions that appear in a large number of channels will only become visible in the final visualization. Thus, any object that may appear in a single channel may be missing from the representation. Also, the spatial information of the object is not considered in determining salient regions.

In this paper we propose a hyper-spectral image visualization technique based on the information of salient regions both in spatial and spectral domain. We propose to generate the saliency maps using Spectral Residual [3] as it extracts the 
salient regions using both high frequency information and intensity variation in images. Saliency map is extracted from each band of hyper-spectral image and these saliency maps are fused with the corresponding hyper-spectral bands. Next, CMF stretching [4] is employed for generation of three channels from resultant high dimensional hyper-spectral image.

The paper is arranged as follows: In Section 2 we present a brief over view of saliency techniques and their use in visualization of hyper-spectral images. Section 3 presents the proposed visualization method followed by testing results in Section 4.

\section{LITERATURE REVIEW}

\subsection{Visualization of Hyper-spectral Images}

Color Matching Function (CMF), defining a linear combination of three visible wavelength ranges $r(\lambda), g(\lambda)$ and $\mathrm{b}(\lambda)$ was proposed by the Commission Internationale d'Eclairage (ECI) in 1931. For visualization of hyper-spectral images Jacobson et al. [4] stretched CMF beyond the visible spectrum to incorporate the spectral range of hyper-spectral images. In [5], the authors propose to use the stretched CMF technique in the CIE L*a*b* color space. Tyo et al. [6] and Tsagaris et al. [7] proposed PCA for dimensionality reduction of HS images so that a large number of spectral channels can be reduced to three spectral channels. They proposed to select the three Principal Components with the maximum variance for representing the RGB channels. Zhanget et al. [8] investigated the utilization of various color spaces for mapping of Principal and Independent Components to the tristimulus display systems. Other band selection techniques for mapping of spectral bands to tri-stimulus display channels make use of entropy, contrast and spectral derivatives have been presented in [9].

\subsection{Saliency in Images}

The term saliency describes the visually interesting regions in images and hence is generally used as a technique for content-based image indexing and retrieval [10]. The benchmark among saliency detecting approaches is Itti's model [11], which make use of color, intensity and orientation information to create a saliency map. In another approach Achanta et al. [12] use the CIE L*a*b* color space for saliency estimation. In [3], the authors propose a Fourier Transform based algorithm called Spectral Residual saliency detection. The proposed algorithm determines the salient regions by analyzing the log-spectrum of an input image.
Phase Quaternion Fourier Transform (PQFT) [13] based approach uses intensity and color features of an image while using the quaternion Fourier Transform for saliency detection.

The above-presented methods can detect saliency in either gray scale or in three channel colored images and cannot be directly used on HS images.

\subsection{Saliency in Hyper-spectral Images}

Initially, Le Moan et al. [14] proposed a method based on saliency for automatic identification objects of interest to enhance the separation between the foreground and background during the dimensionality reduction process. Improving upon their method they have recently proposed portioning of bands in three groups and each group is processed separately to obtain three saliency maps [2]. These saliency maps are combined together to get RGB image. However, the non-salient regions appear dark and color consistency is not addressed

\section{METHODOLOGY}

We propose to calculate salient regions in each band of the hyper-spectral image using Spectral Residual (SR) Algorithm [3] and PQFT [13]. One advantage of selecting SR based saliency estimation is that it is computationally efficient and thus quickly calculates salient regions in each spectral band while PQFT uses both spatial and spectral information for detection of salient regions. Next the saliency maps are fused with the hyper-spectral image so that salient regions gets more weightage and become prominent after dimensionality reduction through CMF stretching. We propose two different techniques for fusion of saliency maps with hyper-spectral images. The two proposed techniques are described below.

\subsection{Algorithm 1: Dual CMF Fusion}

We propose to perform saliency detection on each band of HS image and then apply CMF stretching using Stiles-Burch $10^{\circ}$ CMF [15] resulting in a 3 channel (SB-CM-HS) image. The advantage of using Stiles-Burch $10^{\circ} \mathrm{CMF}$ is that it gives more weightage to bands which will be represented in Red color spectrum i.e. the infra-red bands of the HS image, thus explicitly enhancing a particular part of the HS spectrum. Also, another tri-color composite is generated through CMF stretching without saliency detection called (CM-HS) image. Respective channels (ch) of the two images are fused together to get the final representation as: 

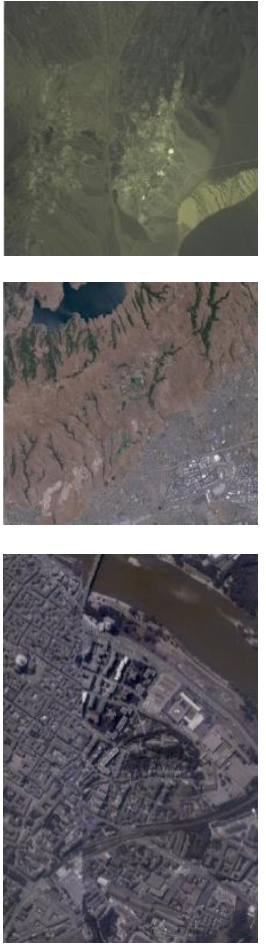

a
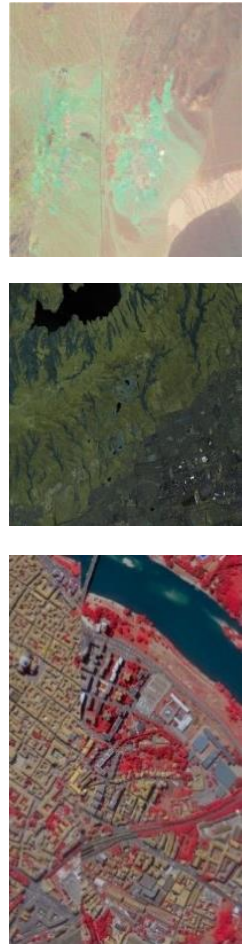

b
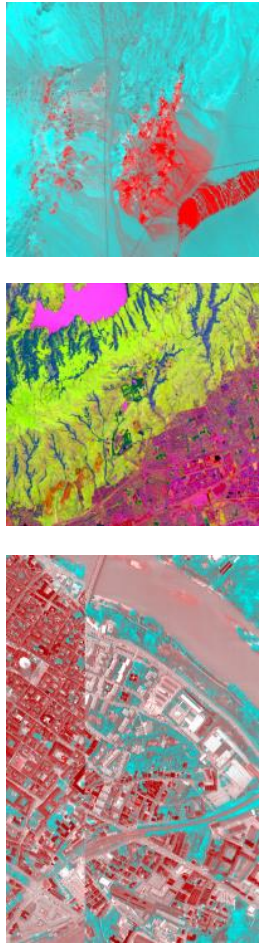

C
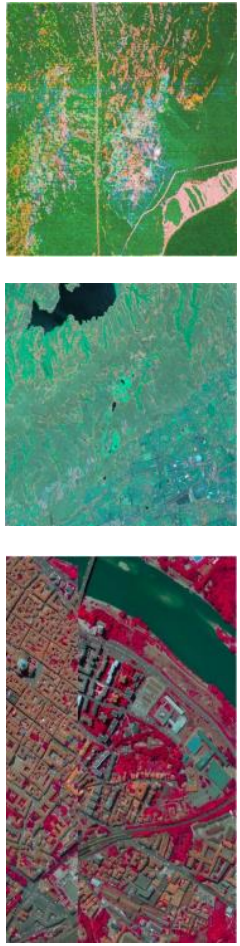

d
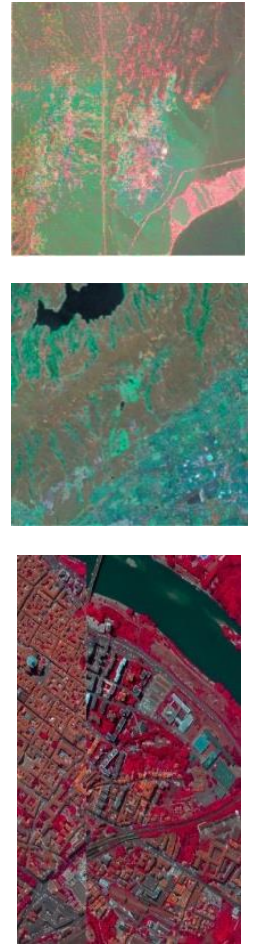

e

Fig. 1: Row-wise: AVIRIS Cuprite, AVIRIS Moffett, ROSIS Pavia Centre.

Columns from left to right: True Color Composite [4], Pseudo Color Composite, PCA based representation [6], Dual CMF Fusion, PCA \&Saliency Fusion

$$
\begin{gathered}
\mathrm{RGB}(\mathrm{ch})=\alpha(\mathrm{ch}) * \mathrm{SB}-\mathrm{CM}-\mathrm{HS}(\mathrm{ch})+\beta(\mathrm{ch}) * \mathrm{CM} \\
-\mathrm{HS}(\mathrm{ch}))
\end{gathered}
$$

Where $\alpha, \beta$ for the red channel can be calculated as: if,

$$
\begin{gathered}
\frac{\rho(S B-C M(r), S B-C M(g))}{\rho(C M(r), C M(g))} \leq 1 \\
\beta(r)=\rho(\mathrm{SB}-\mathrm{CM}(\mathrm{r}), \mathrm{SB}-\mathrm{CM}(\mathrm{g})) / \rho(\mathrm{CM}(\mathrm{r}), \mathrm{CM}(\mathrm{g})) \\
\alpha(\mathrm{r})=1-\beta(\mathrm{r})
\end{gathered}
$$

else

$$
\begin{gathered}
\alpha(r)=\rho(\mathrm{SB}-\mathrm{CM}(\mathrm{r}), \mathrm{SB}-\mathrm{CM}(\mathrm{g})) / \rho(\mathrm{CM}(\mathrm{r}), \mathrm{CM}(\mathrm{g})) \\
\beta(\mathrm{r})=1-\alpha(\mathrm{r})
\end{gathered}
$$

$\rho$ represents correlation. The same procedure should be repeated for the green and blue channels.

\subsection{Algorithm 2: PCA \& Saliency Fusion}

To emphasize more on the salient regions in hyper-spectral image, we divide the HS in 3 equal parts i.e. if there are 90 bands then each part has 30 bands. PCA is applied on each part and $1^{\text {st }}$ Principal Component is selected from each part to form an RGB image. PQFT [13] is applied on this image to get a single channel saliency map ( $\mathrm{SM}_{\mathrm{PCA}}$ ). This saliency map is added with each hyper-spectral band along with the corresponding saliency map obtained through Spectral Residual method for each HS band. CMF stretching is applied on the resultant Fused HS image. Using this scheme, salient regions appear bright as compared to non-salient regions in the final visualization.

$$
\begin{aligned}
& R G B \\
& =C M F\left(\frac{H S I(:,:, N)+\text { Saliency } \operatorname{maps}(:,:, N)+S M_{P C A}}{3}\right) \\
& N \in\{1,2, \ldots, k\}
\end{aligned}
$$

\section{RESULTS AND DISCUSSION}

We have tested the proposed methods (Fig 1(d), (e)) on AVIRIS Cuprite, AVIRIS Moffett field and ROSIS Pavia HS images. For comparison we have presented the true color composite results as Fig 1(a), obtained by selecting the bands in the visible spectral range i.e. from 400 to $700 \mathrm{~nm}$ and applying CMF [4]. Next the results of applying CMF to the entire HS image range are presented as pseudo color composite images Fig 1(b). The results obtained by PCA based visualization scheme of [6] are presented as Fig.1(c). The true color composite results are incapable of showing any 
information from the infrared region because they only represent the visible spectral range. Pseudo-color results give a decent visualization of data however; any object present in a limited region of spectral region will be suppressed and hence will not be visible in the final result. Visualization using PCA based method presented in [6] shows vivid colors and a very saturated image. The PCA bands correspond to the maximum data variance, but mapping those three bands to $R$, $\mathrm{G}$, and $\mathrm{B}$ does not necessarily yield maximum human visual discriminability. Since PCA maps colors without a fixed semantic meaning their colors are not dependent on spectral position. However, the proposed method does not suffer from this shortcoming because colors are assigned on the basis on spectral position of object in hyper-spectral image For the AVIRIS Cuprite and Moffett images, the presented results clearly show variation in geological properties of the area. River is highlighted in Cuprite scene, which is not visible in results obtained by existing CMF based methods. Results of Moffett scene also highlight the geological variations in scene and urban area can be easily distinguished from mountain region in the results produced by the proposed methods. Result from PCA is also able to distinguish but gives distracting colors which make it difficult to recognize that what is type of terrain.

\section{REFERENCES}

[1] A. Yarbus, "Eye Movements and Vision," Plenum Press, 1967.

[2] S. Le Moan, A. Mansouri, J. Hardeberg and Y. Voisin, "Saliency for Spectral Image Analysis," IEEE Journal of Selected Topics in Applied Earth Observations and Remote Sensing, vol. 6, no. 6, pp. 2472-2479, Dec 2013.

[3] Zhang L. and Hou X., "Saliency detection: A spectral residual approach," in IEEE Conference on Computer Vision Pattern Recognition, 2007.

[4] N. Jacobson and M. Gupta, "Design goals and solutions for display of hyperspectral images," IEEE Transactions on Geoscience and Remote Sensing, vol. 43, no. 11, p. 2684-2692, Nov 2005.

[5] S. L. Moan, A. Mansouri, J. Hardeberg and Y. Voisin, "A class separability-based method for multi/hyperspectral image color visualization," in IEEE International Conference on Image Processing, 2010.

[6] J. Tyo, A. Konsolakis, D. Diersen and R. Olsen, "Principal components-based display strategy for spectral imagery," IEEE Tranactions on Geoscience and Remote Sensing, vol. 41, no. 3, p. 708-718, 2003.
[7] V. Anastassopoulos and V. Tsagaris, "Multispectral image fusion for improved RGB representation based on perceptual attributes," International Journal of Remote Sensing, vol. 26, no. 15, p. 3241-3254, 2005.

[8] H. Zhang, D. Messinger and E. Montag, "Perceptual display strategies of hyperspectral imagery based on PCA and ICA," in Proceedings of SPIE, 2006.

[9] B. Demir, A. Celebi and S. Erturk, "A low-complexity approach for the color display of hyperspectral remotesensing images using onebit-transform-based band selection," IEEE Transactions on Geoscience and Remote Sensing, vol. 47, no. 1, p. 97-105, 2009.

[10] K. Vu, K. Hua and W. Tavanapong, "Image retrieval based on regions," IEEE Trans. Knowledge Data Eng, vol. 15, no. 4, p. 1045-1049, Jul-Aug 2003.

[11] L. Itti, C. Koch and E. Niebur, "A model of saliencybased visual attention for rapid scene analysis," EEE Transactions on Pattern Analysis and Machine Intelligence, vol. 20, p. 1254-1259, Nov 1998.

[12] R. Achanta, S. Hemami, F. Estrada and S. Susstrunk, "Frequency-tuned salient region detection," IEEE Conference on Computer Vision and Pattern Recognition CVPR, pp. 1604, 2025, June 2009.

[13] C. Guo and L. Zhang, "A Novel Multiresolution Spatiotemporal Saliency Detection Model and Its Applications in Image and Video Compression," IEEE Transactions on Image Processing, vol. 19, no. 1, pp. 185-198, Jan 2010.

[14] S. L. Moan, F. Deger, A. Mansouri, Y. Voisin and J. Hardeberg, "Salient pixels and dimensionality reduction for display of multi/hyperspectral images," in 5th international conference on Image and Signal Processing ICISP'12, 2012.

[15] W. Burch, S. Stiles and J. M., "N.P.L. colour-matching investigation," Opt. Acta, p. 1-26, 1959. 